\title{
Prevalence and management of hypertension in patients with acute coronary syndrome vary with gender: Observations from the Chinese registry of acute coronary events (CRACE)
}

\author{
YUN LIN, WEIQI PAN, SHANGQIU NING, XIANTAO SONG, ZENING JIN and SHUZHENG LV
}

Department of Cardiology, Beijing Anzhen Hospital Affiliated to Capital Medical University, Beijing 100029, P.R. China

Received December 23, 2012; Accepted March 13, 2013

DOI: $10.3892 / \mathrm{mmr} .2013 .1461$

\begin{abstract}
Hypertension affects one billion people worldwide and is an independent risk factor for death after acute coronary syndrome (ACS). The aim of this study was to examine the prevalence and medical treatment of hypertension among 1,301 ACS patients enrolled into the Chinese registry of acute coronary events (CRACE) trial. Analyses were performed by gender, with both genders combined and according to international practice. Multivariable models identified factors associated with use of different classes of antihypertensive medication, and examined the correlation between hypertension and gender with mortality. The use of angiotensin-converting enzyme inhibitors (ACEI), $\beta$-blockers, calcium channel blockers (CCBs) and diuretics increased in both genders during management of presenting ACS. Hypertensive men were more likely to have been receiving $\beta$-blockers when they were discharged (77.2\%) than women (69.2\%). Hypertensive women were more likely to have received diuretics when they were discharged $(28.4 \%)$ than men $(22 \%)$. ACEI use increased by $\sim 60 \%$ (absolute increase) in both women and men as a result of ACS treatment, but remained similar between the genders, and the same phenomenon was observed in the use of CCBs. Moreover, hypertensive women were less likely to receive evidence-based medication to treat their acute coronary event than men (for women and men, respectively: $\beta$-blocker, 69.2 vs. $77.2 \%$; ACEI, 85.8 vs. $87.5 \%$ ). Hypertension is more prevalent in women than in men with ACS, and its medical management varies with gender, but it has a similar association with mortality in both genders. Opportunities exist to improve medical therapy and outcomes for women with hypertension.
\end{abstract}

Correspondence to: Dr Shuzheng Lv, Department of Cardiology, Beijing Anzhen Hospital Affiliated to Capital Medical University, 2 Anzhen Road, Chaoyang District, Beijing 100029, P.R. China E-mail: shuzh_lv@163.com

Key words: hypertension, sex, acute coronary syndromes, $\beta$-blockers

\section{Introduction}

According to the World Health Organization, hypertension affects one billion people worldwide and is a major risk factor for inducing all forms of cardiovascular disease, exerting a similar effect in both women and men $(1,2)$. Hypertension is a risk factor for death and ischemic events after the occurrence of acute coronary syndrome (ACS) (3-6). Cardiovascular disease is the most frequent cause of mortality in both genders; however, in the past 20 years, cardiovascular mortality has decreased in men but increased in women $(7,8)$. Demographic statistics reveal that hypertension is more common in men than women at a younger age, but in the 45-54 year age group, it is more prevalent in women. Hypertension is estimated to affect over a quarter of all women in the USA and this increases markedly with age; only $\sim 3-4 \%$ of women $<35$ years have hypertension, but $75 \%$ of women $>75$ years are affected (9). Despite this, $19 \%$ of women with hypertension do not recognize that is a risk factor for cardiovascular disease, and as many as $75 \%$ may not be adequately treated $(10,11)$. In addition to being a risk factor for epicardial coronary disease, hypertension leads to microcirculatory dysfunction, which disproportionately affects women, with significant morbidity (12-16).

\section{Materials and methods}

Patient selection. A total of 12 tertiary hospitals across China participated in the Chinese registry of acute coronary events (CRACE) trial. The selected patients represented populations with different demographic characteristics. Data collection began with 1,301 ACS patients between January 2001 and October 2003.

At each hospital, a trained coordinator collected data on the following, using a standard case report form: patient demographic and clinical characteristics, medical history, duration of pre-hospital delay, symptoms, electrocardiographic findings, use of cardiac medications and interventional procedures, and hospital-associated outcomes. A standardized set of definitions for patient-related variables and clinical diagnosis were used.

Inclusion and exclusion criteria. Patients needed to be at least 18 years old, alive at the time of presentation, hospitalized with a presumed diagnosis of ACS and have at least one of the 
Table I. Baseline characteristics of patients with and without hypertension.

\begin{tabular}{|c|c|c|c|c|}
\hline \multirow[b]{2}{*}{ Characteristics } & \multicolumn{2}{|c|}{ Hypertension } & \multicolumn{2}{|c|}{ No hypertension } \\
\hline & Female $(n=211)$ & Male $(n=558)$ & Female $(n=107)$ & (Male $n=425$ ) \\
\hline \multicolumn{5}{|l|}{ Demographics } \\
\hline Age, mean $\pm \mathrm{SD}$ & $68.17 \pm 8.49$ & $62.85 \pm 10.43$ & $65.38 \pm 8.48$ & $60.43 \pm 12.06$ \\
\hline \multicolumn{5}{|l|}{ History and risk factors (\%) } \\
\hline Diabetes mellitus & 34.1 & 21.9 & 24.3 & 14.4 \\
\hline Hypercholesterolemia & 36.5 & 32.6 & 22.4 & 22.8 \\
\hline Current cigarette smoking & 6.6 & 66.3 & 6.5 & 66.1 \\
\hline Prior MI & 13.3 & 14.9 & 11.2 & 13.4 \\
\hline Prior stroke & 12.3 & 12.2 & 3.7 & 6.4 \\
\hline Congestive heart failure & 8.5 & 6.5 & 7.5 & 4.7 \\
\hline Prior PCI & 6.2 & 3.6 & 0.9 & 2.8 \\
\hline Prior CABG & 0 & 0.7 & 0.9 & 0 \\
\hline \multicolumn{5}{|l|}{ Clinical presentation } \\
\hline $\mathrm{BMI}, \mathrm{kg} / \mathrm{m}^{2}$ & $24.74 \pm 3.76$ & $25.15 \pm 7.12$ & $25.07 \pm 3.09$ & $24.61 \pm 5.97$ \\
\hline Killip $\geq$ III & 10.4 & 11.3 & 11.2 & 14.6 \\
\hline Creatinine clearance, $\mathrm{ml} / \mathrm{min}$ & $74.57 \pm 44.59$ & $80.70 \pm 57.69$ & $80.43 \pm 52.5$ & $80.83 \pm 50.81$ \\
\hline $\mathrm{SBP}, \mathrm{mmHg}$ & $137.32 \pm 22.96$ & $136.22 \pm 26.64$ & $126.98 \pm 23.12$ & $118.32 \pm 19.68$ \\
\hline $\mathrm{DBP}, \mathrm{mmHg}$ & $79.61 \pm 15.85$ & $81.58 \pm 15.19$ & $75.99 \pm 14.07$ & $72.97 \pm 13.67$ \\
\hline \multicolumn{5}{|l|}{ Type of ischemic events (\%) } \\
\hline STEMI & 41.2 & 45 & 34.6 & 53.2 \\
\hline NSTEMI & 19.4 & 16.8 & 15.9 & 15.3 \\
\hline UAP & 39.3 & 38.2 & 49.5 & 31.5 \\
\hline
\end{tabular}

MI, myocardial infarction; PCI, percutaneous coronary intervention; CABG, coronary artery bypass graft; SBP, systolic blood pressure; DBP, diastolic blood pressure; STEMI, ST elevated myocardial infarction; NSTEMI, non-ST elevated myocardial infarction ; UAP, unstable angina pectoris.

following: electrocardiographic changes consistent with ACS, elevated serum biochemical markers for cardiac necrosis or documented evidence of coronary artery disease (CAD). To qualify, the ACS must not have been precipitated or accompanied by a significant comorbidity such as trauma or surgery. Where informed consent could not be obtained, patients were excluded from the study (5).

Quality control. The 12 triple class hospitals involved in this study are teaching hospitals affiliated with medical universities in different regions of China. To eliminate patient selection bias, each hospital enrolled the first 5 ACS patients to be hospitalized each month. Completed case report forms were faxed to the data management organization, where they were reviewed for completeness and clinical validity. Resulting queries were referred back to the originating site before the forms were processed. The data were scanned into an electronic database and checked manually prior to statistical analysis.

Statistical analysis. Statistical analyses were performed using SPSS 11.0 for PC. Values were expressed as the means \pm SD for continuous variables. Comparisons between different groups were made using a two-tailed Student's t-test for continuous variables and by Pearson's Chi-square test and Fisher's exact test for binary variables. Differences were considered to indicate statistical significance when $\mathrm{P}<0.05$.

\section{Results}

Patient population. In the CRACE trial the prevalence of hypertension was $66.4 \%$ among female patients with ACS and $56.8 \%$ among male patients. Fig. 1 shows the prevalence of hypertension of different age groups. Unlike observations in unselected populations, among the ACS patients in this study, the prevalence of hypertension was greater among women than among men for each decile of age. As shown in Fig. 2, the age distribution among hypertensive women was skewed toward older ages, but age was found to be more regularly distributed among hypertensive men. Overall, for both genders, patients with hypertension were older and more often had diabetes, hypercholesterolemia, prior incidence of myocardial infarction (MI), prior incidence of stroke, and history of congestive heart failure. Additionally, female patients were more likely to present with MI rather than unstable angina pectoris (UAP) compared to female patients without hypertension (Table I).

When comparing men and women with hypertension, the women were older, had lower estimated creatinine clearance, 
Table II. Use of antihypertensive medications among people with hypertension before and after presentation with ACS.

\begin{tabular}{lccc}
\hline & $\begin{array}{c}\text { Female } \\
(\mathrm{n}=211)\end{array}$ & $\begin{array}{c}\text { Male } \\
(\mathrm{n}=558)\end{array}$ & P-value \\
\hline BP control at admission (\%) & & & \\
SBP <140 mmHg & 61.1 & 64.2 & \\
DBP <90 mmHg & 82 & 78 & \\
Before presentation (\%) & & & \\
ACE inhibitors & 22.7 & 21.5 & 0.671 \\
$\beta$-blocker & 17.5 & 14.9 & 0.029 \\
CCBs & 15.2 & 14.7 & 0.801 \\
Diuretics & 4.3 & 3.9 & 0.004 \\
At discharge $(\%)$ & & & \\
ACE inhibitors & 85.8 & 87.5 & 0.524 \\
$\beta$-blocker & 69.2 & 77.2 & 0.013 \\
CCBs & 35.1 & 34.6 & 0.878 \\
Diuretics & 28.4 & 22 & 0.020 \\
\hline
\end{tabular}

SBP, systolic blood pressure; DBP, diastolic blood pressure; ACE, angiotensin converting enzyme and $\mathrm{CCBs}$, calcium channel blockers .

Table III. Reperfusion therapies and outcomes between in-hospital female and male ACS patients with hypertension.

\begin{tabular}{lccc}
\hline Outcomes & $\begin{array}{c}\text { Female } \\
(\mathrm{n}=211)\end{array}$ & $\begin{array}{c}\text { Male } \\
(\mathrm{n}=558)\end{array}$ & P-value \\
\hline Reperfusion therapies (\%) & & & $<0.0001$ \\
$\quad$ No reperfusion & 48.8 & 31.4 & \\
PCI & 44.1 & 54.3 & \\
Thrombolysis & 2.8 & 6.5 & \\
Others & & 7.8 & \\
In-hospital MACCEs (\%) & 4.3 & & \\
MI within 24 h & 4.7 & 3.4 & 0.08 \\
Recurrent angina & 27 & 15.4 & $<0.0001$ \\
Congestive heart failure & 16.1 & 10.8 & 0.008 \\
Stroke & 0.9 & 0.6 & 0.156 \\
Bleeding & 1.4 & 1.1 & 0.585 \\
Discharge status (\%) & & & 0.669 \\
Death & 3.8 & 3.6 & \\
Go home & 23.3 & 21.9 & \\
\hline
\end{tabular}

ACE, angiotensin-converting enzyme; LMWH, low molecular weight heparin; MACCEs, major adverse cardiovascular cerebral events. P-value represent no reperfusion versus reperfusion (PCI, thrombolysis) therapy between the two groups. ${ }^{\mathrm{a} O t h e r s ~ i n d i c a t e d ~ t h e r a p i e s ~}$ including $\mathrm{CABG}$ and $\mathrm{PCI}+\mathrm{CABG}$.

and were more likely to have diabetes, hypercholesterolemia and prior congestive heart failure (Table I). They tended to smoke less and were less likely to have had a prior incidence of MI or to present with MI as their index event. Median SBP at presentation was higher $(1 \mathrm{mmHg})$ among women with

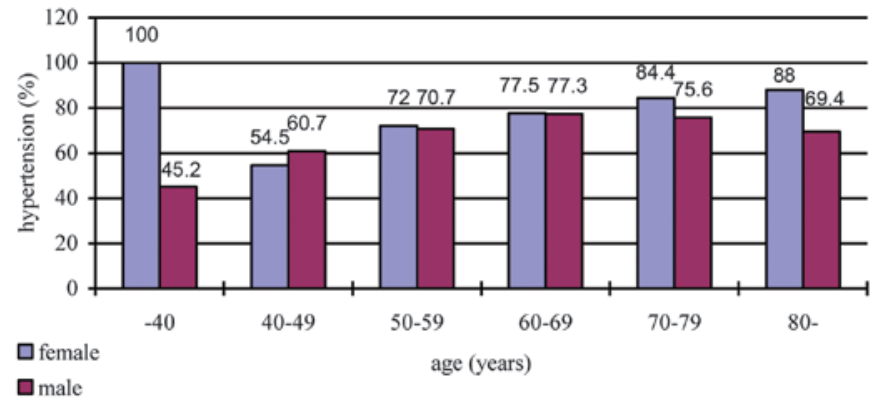

Figure 1. Prevalence of hypertension by deciles of age among Chinese women and men with ACS. ACS, acute coronary syndrome.

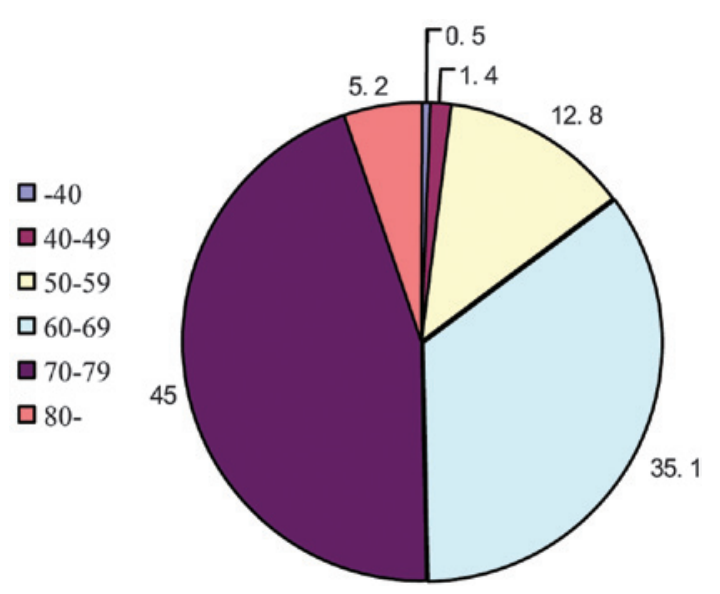

Figure 2. Age distribution of hypertension among Chinese women and men with prevalent hypertension and ACS. ACS, acute coronary syndrome.

hypertension than men, despite the use of significantly more antihypertensive medications (Table I).

Antihypertensive medication use. As shown in Table II, more female ACS patients with hypertension were well controlled than male patients. The use of diuretics and $\beta$-blockers was found to be higher in hypertensive women, yet no difference was found with regards to the use of angiotensin-converting enzyme inhibitors (ACEI) and calcium channel blockers (CCBs) between the two groups. The use of all medications including ACEI, $\beta$-blockers, CCBs and diuretics increased in both genders during management of the presenting ACS, but, when discharged, hypertensive men $(77.2 \%)$ were more likely to have been receiving $\beta$-blockers than women (69.2\%). Although at discharge, the rate of diuretic use increased by $\sim 20 \%$ in both genders, hypertensive women were more likely to have been receiving diuretics $(28.4 \%)$ compared to men (22\%). ACEI use increased by $\sim 60 \%$ (absolute increase) in both women and men as a result of ACS treatment, but use remained similar between the genders, and the same phenomenon was observed in the use of CCBs.

Outcomes among hypertensive women and men. Men with hypertension were more likely to receive reperfusion therapies during hospitalization than hypertensive women (Table III). Although no difference was found regarding the in-hospital death rate between genders, female patients experienced 
more in-hospital composite endpoints, including recurrent angina and congestive heart failure. Despite their higher rate of adverse clinical outcomes, hypertensive women were less likely to receive evidence-based medication to treat their acute coronary event than men (for women and men, respectively: $\beta$-blocker, 69.2 vs. $77.2 \%$; ACEI, 85.8 vs. $87.5 \%$ ).

\section{Discussion}

Among patients with ACS in the CRACE trial, $>50 \%$ had hypertension, and it was found to be more prevalent among women. The association of hypertension with adverse clinical outcomes in these patients was similar in both genders. Among the studied patients with hypertension, $\sim 40 \%$ had failed to maintain their blood pressure at a safe level at the beginning of the study.

As expected, the overall prevalence of hypertension among ACS patients was higher than that which had been reported in the general population. Contrary to observations made regarding the general population, in which the prevalence of hypertension is greater among men than among women at younger ages and the opposite is observed at older ages, we found that the prevalence of hypertension among ACS patients was greater in women than in men in every decile of age. We cannot find a reasonable explanation for this finding, but we suspect that an overall greater burden of risk factors is required to overcome the protective effect of the premenopausal state on the development of CAD among younger women. Alternatively, this may reflect a correlation with other medication use (such as oral contraceptives) that influences both the incidence of hypertension and the occurrence of ischemic events in women compared with men (17).

In our study, hypertensive women were treated with more antihypertensive agents than men, and the median SBP at presentation in women was $1 \mathrm{mmHg}$ higher. The effect of antihypertensive agents on lowering blood pressure has yet to be well studied in each gender, but previous studies have indicated that differences in salt sensitivity, hormonal variation and vascular reactivity may affect hypertension in women (18-21).

We observed that only $61 \%$ of women and $64 \%$ of men with prevalent hypertension at presentation with ACS were well controlled. Observations made from the NHANES III study may reflect this finding in the general population in that NHANES III demonstrated that only $70 \%$ of hypertensive participants were aware of their diagnosis and that $31 \%$ of participants had controlled hypertension (22). Even with close monitoring in the setting of a large-scale randomized trial of hypertension treatment, the 5-year follow-up data from ALLHAT revealed that only $66 \%$ of participants achieved target levels of hypertension control (23). In conclusion, these figures emphasize the need for more active management, particularly among high-risk ACS patients, and suggest that factors other than blood pressure are important in preventing the clinical consequences of hypertension. These additional factors may be associated with the pleiotropic effects of many contemporary antihypertensive medications.

Although the ALLHAT results suggest that diuretics may be more beneficial to prevent primary coronary heart disease than an ACEI, at the time of the SYMPHONY studies, the
American Heart Association prevention guidelines for women with hypertension recommended the use of $\beta$-blockers and ACEI for risk factor modification (24-26). Furthermore, the use of diuretics at discharge after presentation with ACS remained greater in women than in men, with $\beta$-blockers prescribed less often in women. Although we adjusted for comorbid conditions (diabetes, heart failure, and prior MI or bypass surgery) that may have influenced the decision to use a given antihypertensive agent, we could not account for possible unmeasured factors leading to this association. For example, we noted that among the studied patients, women had no epicardial CAD more often than men and more frequently had preserved left ventricular function. This may be related to gender differences in microcirculatory dysfunction associated with hypertension and other comorbid diseases, resulting in a higher incidence of women presenting with ACS despite having no epicardial CAD (27-30), and it may account for gender-related differences with regard to medication given upon discharge.

Despite the higher prevalence of hypertension in women than men with ACS in our study, cardiovascular outcomes were similar after adjusting for age and comorbidities. Furthermore, similar results were found in the overall population of the SYMPHONY trials: the association of hypertension with adverse clinical outcomes was similar in the two genders.

Thus, hypertensive female patients are a vital target population for active evidence-based primary and secondary risk factor modification. A study of the correlation between gender, hypertension and outcomes in the NHANES III revealed that preventable population-attributed risk of coronary heart disease events was higher in women than men. From this observation, it was estimated that optimal control of blood pressure could prevent more than one-third of coronary heart disease events in men and more than half of the events in women. We observed that hypertensive women were less likely to receive evidence-based medications for ACS during hospitalization than hypertensive men. This finding suggests a need for continued education on the high risk of adverse outcomes in hypertensive women presenting with ACS and the importance of using therapies shown to attenuate that risk, such as $\beta$-blockers and ACEI (1).

This study observed the differences in managing hypertension between male and female ACS patients. As is inherent in such analyses, selection bias may exist, and detailed data about the duration and control of hypertension prior to admission were not collected. Therefore, our observations, including age and prevalence distributions, may not represent the entire population of ACS patients, nor are they intended to reflect an unselected population with hypertension. Results should be interpreted with caution due to the fact that examinations of gender and hypertension were not specified as a priori hypotheses in the clinical trial design.

In conclusion, this study revealed gender-related differences in the prevalence and treatment of hypertension among patients with ACS. It demonstrated the relationship between gender, hypertension and outcomes in this population and highlighted the greater prevalence of hypertension between women and men with ACS and suggests more active treatment is required for women with hypertension. 


\section{References}

1. World Health Organization: The world health report 2002: reducing risks, promoting healthy life. http://www.who.int/ whr/2002. Accessed January 2005.

2. Yusuf S, Hawken S, Ounpuu S, et al: Effect of potentially modifiable risk factors associated with myocardial infarction in 52 countries (the INTERHEART study): case-control study. Lancet 364: 937-952, 2004.

3. Lee KL, Woodlief LH, Topol EJ, et al: Predictors of 30-day mortality in the era of reperfusion for acute myocardial infarction. Results from an international trial of 41,021 patients. GUSTO-I Investigators. Circulation 91: 1659-1668, 1995.

4. Newby LK, Bhapkar MV, White HD, et al: Predictors of 90-day outcome in patients stabilized after acute coronary syndromes. Eur Heart J 24: 172-181, 2003.

5. Antman EM, Cohen M, Bernink PJ, et al: The TIMI risk score for unstable angina/non-ST elevation MI: a method for prognostication and therapeutic decision making. JAMA 284: 835-842, 2000.

6. Pepine CJ, Handberg EM, Cooper-DeHoff RM, et al: A calcium antagonist vs a non-calcium antagonist hypertension treatment strategy for patients with coronary artery disease The International Verapamil-Trandolapril Study (INVEST): a randomized controlled trial. JAMA 290: 2805-2816, 2003.

7. AHA, heart disease and stroke statistics - 2005 update. American Heart Association, Dallas, TX, USA, 2004

8. Chobanian AV, Bakris GL, Black HR, et al: Seventh report of the Joint National Committee on Prevention, Detection, Evaluation, and Treatment of High Blood Pressure. Hypertension 42: 12061252, 2003.

9. Wolz M, Cutler J, Roccella EJ, et al: Statement from the National High Blood Pressure Education Program: prevalence of hypertension. Am J Hypertens 13: 103-104, 2000.

10. Vasan RS, Beiser A, Seshadri S, et al: Residual lifetime risk for developing hypertension in middle-aged women and men: The Framingham Heart Study. JAMA 287: 1003-1010, 2002.

11. Rosenthal T and Oparil S: Hypertension in women. J Hum Hypertens 14: 691-704, 2000.

12. Reis SE, Holubkov R, Conrad Smith AJ, et al: Coronary microvascular dysfunction is highly prevalent in women with chest pain in the absence of coronary artery disease: results from the NHLBI WISE study. Am Heart J 141: 735-741, 2001.

13. Rosengren A, Wallentin L, Gitt AK, et al: Sex, age, and clinical presentation of acute coronary syndromes. Eur Heart J 25: 663-670, 2004

14. Gulli G, Cemin R, Pancera P, et al: Evidence of parasympathetic impairment in some patients with cardiac syndrome $\mathrm{X}$. Cardiovasc Res 52: 208-216, 2001

15. Iriarte M, Caso R, Murga N, et al: Microvascular angina pectoris in hypertensive patients with left ventricular hypertrophy and diagnostic value of exercise thallium-201 scintigraphy. Am J Cardiol 75: 335-339, 1995.

16. Schwartzkopff B, Motz W, Frenzel H, et al: Structural and functional alterations of the intramyocardial coronary arterioles in patients with arterial hypertension. Circulation 88: 993-1003, 1993.
17. Jaffe LS and Seely EW: The heterogeneity of the blood pressure response to hormonal contraceptives. Curr Opin Endocrinol Diabetes 2: 257-265, 1995.

18. Appel LJ, Champagne CM, Harsha DW, et al: Effects of comprehensive lifestyle modification on blood pressure control: main results of the PREMIER clinical trial. JAMA 289: 2083-2093, 2003.

19. Staessen J, Bulpitt CJ, Fagard R, et al: The influence of menopause on blood pressure. J Hum Hypertens 3: 427-433, 1989.

20. McIntyre M, Hamilton CA, Rees DD, et al: Sex differences in the abundance of endothelial nitric oxide in a model of genetic hypertension. Hypertension 30: 1517-1524, 1997.

21. Duvernoy C, Martin J, Briesmiester K, et al: Myocardial blood flow and flow reserve in response to hormone therapy in postmenopausal women with risk factors for coronary disease. J Clin Endocrinol Metab 89: 2783 -2788, 2004.

22. Hajjar I and Kotchen TA: Trends in prevalence, awareness, treatment, and control of hypertension in the United States, 1988-2000. JAMA 290: 199-206, 2003.

23. Cushman WC, Ford CE, Cutler JA, et al: Success and predictors of blood pressure control in diverse North American settings: the antihypertensive and lipid-lowering treatment to prevent heart attack trial (ALLHAT). J Clin Hypertens (Greenwich) 4: 393-404, 2002

24. Mosca L, Manson JE, Sutherland SE, et al: Cardiovascular disease in women: a statement for healthcare professionals from the American Heart Association. Writing Group. Circulation 96: 2468-2482, 1997.

25. Mukherjee D, Fang J, Chetcuti S, et al: Impact of combination evidence-based medical therapy on mortality in patients with acute coronary syndromes. Circulation 109: 745-749, 2004.

26. Fox KA, Goodman SG, Anderson FA Jr, et al: From guidelines to clinical practice: the impact of hospital and geographical characteristics on temporal trends in the management of acute coronary syndromes. The Global Registry of Acute Coronary Events (GRACE). Eur Heart J 24: 1414-1424, 2003.

27. Hochman JS, McCabe CH, Stone PH, et al: Outcome and profile of women and men presenting with acute coronary syndromes: a report from TIMI IIIB. TIMI Investigators. Thrombolysis in Myocardial Infarction. J Am Coll Cardiol 30: 141-148, 1997.

28. The Global Use of Strategies to Open Occluded Coronary Arteries (GUSTO) IIb Investigators: A comparison of recombinant hirudin with heparin for the treatment of acute coronary syndromes. N Engl J Med 335: 775-782, 1996.

29. Becker RC, Terrin M, Ross R, et al: Comparison of clinical outcomes for women and men after acute myocardial infarction. The Thrombolysis in Myocardial Infarction Investigators. Ann Intern Med 120: 638-645, 1994.

30. Tunstall-Pedoe H, Morrison C, Woodward M, et al: Sex differences in myocardial infarction and coronary deaths in the Scottish MONICA population of Glasgow 1985 to 1991. Presentation, diagnosis, treatment, and 28-day case fatality of 3991 events in men and 1551 events in women. Circulation 93: 1981-1992, 1996. 